\title{
Coherent Control of Rare-Earth Ions in On-Chip Devices for Microwave-to-Optical Transduction
}

\author{
John G. Bartholomew ${ }^{1,2}$, Jake Rochman ${ }^{1,2}$, Jonathan M. Kindem ${ }^{1,2}$, Andrei \\ Ruskuc $^{1,2}$, Ioana Craiciu ${ }^{1,2}$, Mi Lei ${ }^{1,2}$, Tian Zhong ${ }^{1,2,3}$, and Andrei Faraon ${ }^{1,2}$ \\ ${ }^{1}$ Kavli Nanoscience Institute and Thomas J. Watson, Sr., Laboratory of Applied Physics, \\ California Institute of Technology, Pasadena, California 91125, USA. \\ ${ }^{2}$ Institute for Quantum Information and Matter, \\ California Institute of Technology, Pasadena, California 91125, USA. \\ ${ }^{3}$ Current address: Institute of Molecular Engineering, University of Chicago, Chicago, Illinois 60637, USA \\ john.g.bartholomew@gmail.com
}

\begin{abstract}
Entangling microwave and optical photons is essential to harness disparate technologies for building larger scale quantum networks. We demonstrate coherent microwave-to-optical transduction using a nanobeam waveguide containing rare-earth ions in a dilution refrigerator. (c) 2019 The Author(s)
\end{abstract}

OCIS codes: $270.1670,160.5690$.

\section{Introduction}

Quantum technologies offer a radically different way of approaching solutions to some of the most challenging questions in science and industry. For quantum technologies to realize these solutions, the quality and scale of entangled qubit networks must continue to grow. To promote such growth, quantum networks will need to capitalize on application-specific hardware, but many quantum hardware systems operate in disparate regimes. For example, many superconducting or spin qubit systems operating in the microwave regime do not have access to the long-distance remote entanglement operations possible with optical photons at room temperature.

Microwave-to-optical (M2O) transduction schemes seek to create quantum interconnects to optically entangle remote microwave-regime qubits. One such scheme is to use an ensemble of rare-earth ions (REIs) in a crystal as a high-efficiency magneto-optical transducer [1]. Here we present a miniaturized architecture for this scheme that achieves coherent transduction of photons from microwave frequencies $\omega_{m}=0.5-12 \mathrm{GHz}$ to the optical domain $\omega_{o} \approx 3 \times 10^{5} \mathrm{GHz}(984.5 \mathrm{~nm})$. Although the current on-chip device (see Figure 1) operates at low efficiency, it serves as a valuable test platform for engineering the REI material and architecture for the next generation of higher performance devices.

(a)

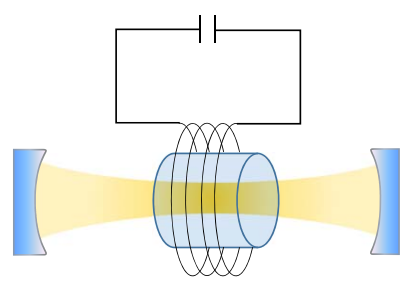

(b)

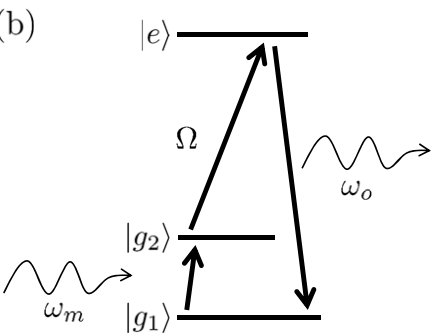

$(\mathrm{c})$

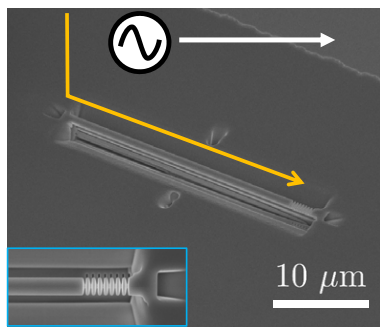

Fig. 1. (a) Concept of the REI magneto-optical transducer. A microwave resonator couples inductively to the spin transition of a REI ensemble in a crystal. The same REIs simultaneously couple to an optical cavity through their transition to an excited state. (b) Energy level diagram for the transduction scheme. An input microwave signal at frequency $\omega_{m}$ is coupled to the spin transition $\left|g_{1}\right\rangle \leftrightarrow\left|g_{2}\right\rangle$. An optical pump with Rabi frequency $\Omega$ coherently upconverts the input to an optical output at frequency $\omega_{o}$. (c) The SEM image shows a waveguide that has been milled, using a focused ion beam, within a coplanar waveguide on the surface of a ${ }^{171} \mathrm{Yb}^{3+}: \mathrm{YVO}_{4}$ substrate. The optical pump is launched into the device through a $45^{\circ}$ coupler, and a photonic crystal mirror at the opposite end (see inset) reflects the optical signal to allow collection from the input coupler. 


\section{Experimental method}

A gold coplanar waveguide (CPW) was fabricated onto the ${ }^{171} \mathrm{Yb}: \mathrm{YVO}_{4}$ substrate by electron-beam lithographic patterning. Suspended nanobeam waveguides and photonic crystal cavities were then milled within the CPW structure using focused ion beam. The devices were mounted in a closed cycle cryostat at $\approx 4 \mathrm{~K}$ or on the mixing chamber of a dilution refrigerator at a base temperature of $\approx 20 \mathrm{mK}$. The properties of the device were probed through optical absorption and coherence measurements, and optically detected magnetic resonance spectroscopy. M2O transduction was detected using Raman heterodyne ( $\mathrm{RH}$ ) spectroscopy [2]. The $\mathrm{RH}$ measurements enable a characterization of the magnetic field and coherent driving conditions for optimal $\mathrm{M} 2 \mathrm{O}$ transduction, as well as a precise confirmation of the spin structure of the $\mathrm{Yb}$ ensemble.

\section{Results}

Figure 2 shows an example of coherent $\mathrm{M} 2 \mathrm{O}$ transduction mediated by ${ }^{171} \mathrm{Yb}$-ions in the $30 \mu \mathrm{m}$-long waveguide shown in Figure 1(c). In this case, the oscillating magnetic field from the CPW couples to the ground-state spin transition at $\approx 675 \mathrm{MHz}$. Transduction from the excited state transition at $3.37 \mathrm{GHz}$ was also measured by using the appropriate V-system.

Although there are advantages to being able to transduce at zero field, including simplified integration with superconducting microwave circuitry, the transduction signal can also be harnessed in applied fields for detailed nuclear magnetic resonance and electron spin resonance characterization of the $\mathrm{Yb}$ ions at dilution fridge temperatures [3]. In our case, operating with waveguides in both the optical and microwave regimes allows continuous mapping of the spin transition properties over a broad frequency range. We will also report on our progress toward pulsed RH measurements of the homogeneous linewidth of the $\mathrm{Yb}$ nuclear and electron spins.
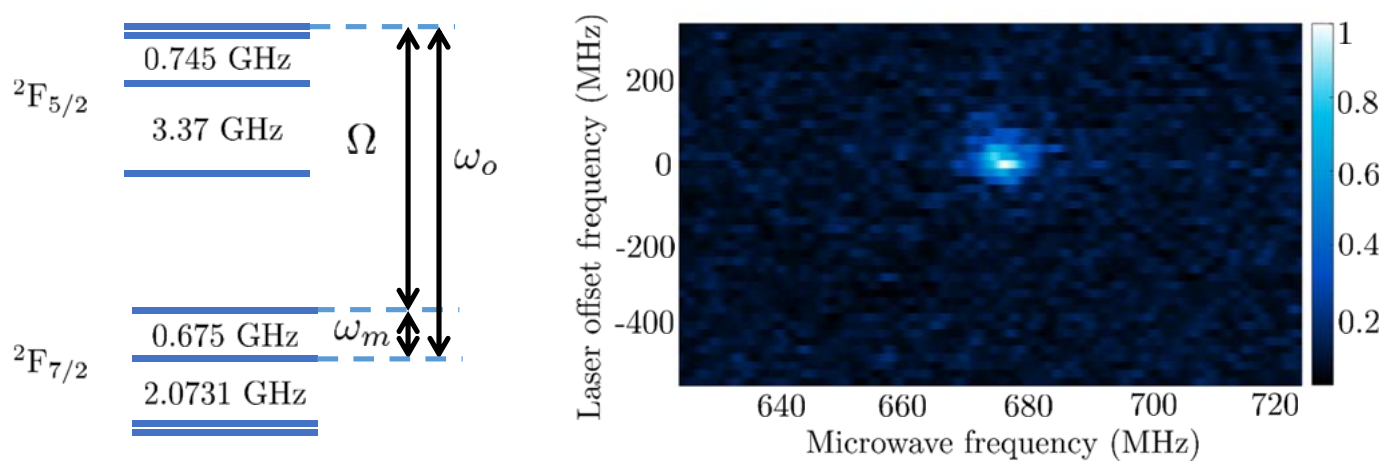

Fig. 2. (left) The energy level structure of ${ }^{171} \mathrm{Yb}$ ions in the $\mathrm{YVO}_{4}$ host at zero field [4], along with the targetted $\Lambda$-system for transduction. (right) An example of a double-resonance RH spectrum showing the normalized upconverted signal (the field strength at $\omega_{o}$ ) as a function of microwave frequency and the frequency offset of the pump laser $(\Omega)$ from the center of the optical transition.

\section{Conclusion}

This realization of combined microwave and optical control of REIs in a nanoscale device is an important step in achieving high efficiency magneto-optical M2O transduction. We will discuss our current efforts to increase the efficiency, and scalability of this architecture beyond this initial demonstration.

\section{References}

1. L. A. Williamson, Y.-H. Chen, and J. J. Longdell, "Magneto-optic modulator with unit quantum efficiency", Phys. Rev. Lett., 113(20), 15 (2014).

2. X. Fernandez-Gonzalvo, Y.-H. Chen, C. Yin, S. Rogge, and J. J. Longdell, "Coherent frequency upconversion of microwaves to the optical telecommunications band in an Er:YSO crystal", Phys. Rev. A, 92(6), 62313 (2015).

3. P. A. Bushev et al., "Ultralow-power spectroscopy of a rare-earth spin ensemble using a superconducting resonator", Phys. Rev. B, 84(6), 060501 (2011).

4. J.M. Kindem et al., "Characterization of ${ }^{171} \mathrm{Yb}: \mathrm{YVO}_{4}$ for photonic quantum technologies", Phys. Rev. B, 98,024404 (2018). 